\title{
Metal-organic framework-based nanocatalytic medicine for chemodynamic therapy
}

\author{
Shutao Gao ${ }^{1,2 \dagger}, \mathrm{Yu} \mathrm{Han}^{2 \dagger}$, Miao Fan ${ }^{2}$, Zhenhua $\mathrm{Li}^{2}$, Kun $\mathrm{Ge}^{2}$, Xing-Jie Liang ${ }^{3}$ and Jinchao Zhang ${ }^{2^{*}}$
}

Chemodynamic therapy (CDT) is an emerging and promising strategy based on the Fenton or Fenton-like reaction in tumors [1-3]. The tumor microenvironment (TME) has the characteristics of low acidity and overexpression of $\mathrm{H}_{2} \mathrm{O}_{2}[4,5]$, thus providing favorable conditions for initiating the Fenton or Fenton-like reaction. In CDT, intratumoral $\mathrm{H}_{2} \mathrm{O}_{2}$ is decomposed into highly toxic hydroxyl radicals $(\cdot \mathrm{OH})$ through the Fenton or Fenton-like reaction catalyzed by a metal-based nanocatalyst, which will cause irreversible damage to DNA, lipids, and proteins [6,7]. TME-based CDT can inhibit tumor growth and reduce the side effects on normal tissues. Recently, many nanocatalytic medicines containing metal elements, such as iron oxide nanoparticles, bimetallic alloys, $\mathrm{MnO}_{2}$, and metal-organic frameworks (MOFs), have been developed for improving the CDT efficiency [6].

MOFs are a type of hybrid porous materials composed of metal ions (clusters) with organic linkers through coordination bonds. Owing to their unique physicochemical properties, such as tunable structure, high porosity, and easy functionalization, MOFs have been extensively used for gas storage/separation [8], sensing [9], catalysis [10], and biomedicine [11]. In particular, the development of nanoscale MOFs increases their potential application in biomedicine, including bioimaging, antimicrobial action, antitumor activity, biosensing, and biocatalysis. Note that, in the past few years, the research of MOFs in CDT has rapidly developed (Fig. 1). This is primarily attributed to the intrinsic structural characteristics. For example, the coordination bonds between the metal ions (clusters) and the organic linkers can be easily broken in the acidic
TME, which releases additional metal ions and provides additional catalysts for CDT [12]. Furthermore, the welldefined pores and excessive porosity enable nanoscale MOFs to act as a carrier for various cargos ranging from diagnostic agents to therapeutic agents. Herein, we highlight the application of MOFs in CDT (Table 1) and hopefully this can promote the development of MOFbased nanocatalytic medicine for CDT.

$\mathrm{Fe}^{2+}$ is a classical Fenton reaction catalyst for the disproportionation of $\mathrm{H}_{2} \mathrm{O}_{2}$ to $\cdot \mathrm{OH}$. CDT efficacy based on the ferrous catalyst is determined by the safe delivery and controllable release of $\mathrm{Fe}^{2+}$ into the tumor. Owing to its excellent acid responsive ability, multiple $\mathrm{Fe}^{2+}$-based MOFs have been developed for CDT. One of the simple and classical designs is from the work reported by RanjiBurachaloo et al. [13]. They developed a folic acid (FA)modified $\mathrm{Fe}^{2+}$-based MOF (rMOF-FA) by reducing $\mathrm{NH}_{2}-$

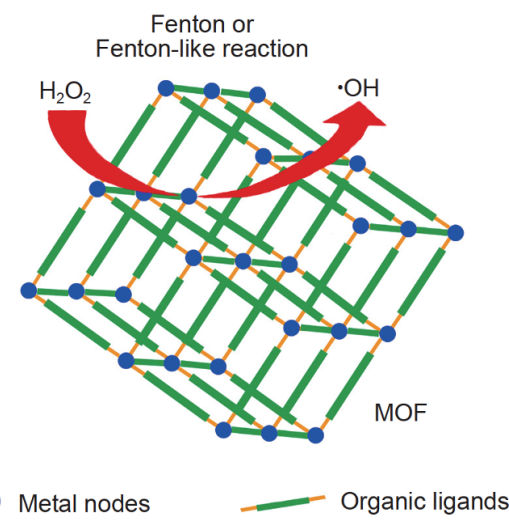

Figure 1 The mechanism for application of MOFs in chemodynamic therapy.

\footnotetext{
${ }^{1}$ College of Science, Hebei Agricultural University, Baoding 071001, China

${ }^{2}$ College of Chemistry\& Environmental Science, Chemical Biology Key Laboratory of Hebei Province, Key Laboratory of Medicinal Chemistry and Molecular Diagnosis of the Ministry of Education, Institute of Life Science and Green Development, Hebei University, Baoding 071002, China

${ }^{3}$ CAS Key Laboratory for Biological Effects of Nanomaterials and Nanosafety, National Center for Nanoscience and Technology, Beijing 100190, China

† These authors contributed equally to this work.

*Corresponding author (email: jczhang6970@163.com)
} 
Table 1 Examples of MOFs-based nanocatalytic medicines

\begin{tabular}{|c|c|c|c|}
\hline Nanocatalytic medicines/MOFs & MOFs' major components & Fenton or Fenton-like catalyst & Ref. \\
\hline $\mathrm{rMOF} / \mathrm{NH}_{2}-\mathrm{MIL}-88 \mathrm{~B}(\mathrm{Fe})$ & $\mathrm{Fe}^{3+}$ and 2-aminoterephthalic acid & $\mathrm{Fe}^{2+}$ & {$[13]$} \\
\hline MD@Lip/MOF-Fe ${ }^{2+}$ & $\mathrm{Fe}^{2+}$ and 2-aminoterephthalic acid & $\mathrm{Fe}^{2+}$ & [14] \\
\hline Co-Fc@GOx/Co-Fc nMOF & $\mathrm{Co}^{2+}$ and $1,1^{\prime}$-ferrocenedicarboxylic acid & $\mathrm{Fe}^{2+}$ & {$[15]$} \\
\hline $\mathrm{Zr}-\mathrm{Fc} \mathrm{MOF}$ & $\mathrm{Zr}^{4+}$ and $1,1^{\prime}$-ferrocenedicarboxylic acid & $\mathrm{Fe}^{2+}$ & {$[16]$} \\
\hline Hf-BPY-Fe/Hf-nMOFs & $\mathrm{Hf}^{4+}$ and 2,2'-bipyridine-5,5'-dicarboxylic acid & $\mathrm{Fe}^{2+}$ & {$[17]$} \\
\hline MGH nanoamplifiers/MIL-100 & $\mathrm{Fe}^{3+}$ and 1,3,5-benzenetricarboxylic acid & $\mathrm{Fe}^{3+}$ & {$[18]$} \\
\hline AMP NRs/MIL-101 & $\mathrm{Fe}^{3+}$ and 1,3,5-benzenetricarboxylic acid & $\mathrm{Fe}^{3+}$ & {$[19]$} \\
\hline DMH NPs/MIL-100 & $\mathrm{Fe}^{3+}$ and 1,3,5-benzenetricarboxylic acid & $\mathrm{Fe}^{3+}$ & [20] \\
\hline $\mathrm{Cu}-\mathrm{TBP}$ nMOF & $\mathrm{Cu}^{2+}$ and tetrakis(4-carboxyphenyl)porphyrin & $\mathrm{Cu}^{2+}$ & {$[21]$} \\
\hline PCN-224(Cu)-GOD@MnO $2 / \mathrm{PCN}-224$ & $\mathrm{Cu}^{2+}$ and tetrakis(4-carboxyphenyl)porphyrin & $\mathrm{Cu}^{+}$ & {$[22]$} \\
\hline CaO2@DOX@ZIF-67/ZIF-67 & $\mathrm{Co}^{2+}$ and 2-methylimidazole & $\mathrm{Co}^{2+}$ & [23] \\
\hline
\end{tabular}

MIL-88B(Fe). After internalization by tumor cells, the asprepared rMOF-FA was decomposed and $\mathrm{Fe}^{2+}$ was released into the lysosome. Then, the released $\mathrm{Fe}^{2+}$ could induce the intracellular Fenton reaction to produce a high concentration of $\cdot \mathrm{OH}$ to destroy tumor cells. However, the $\mathrm{pH}$ value of the lysosome was $\sim 5.0$, which cannot satisfy the requirements of an effective Fenton reaction $(\mathrm{pH}=3.0-4.0)$. Moreover, the limited concentration of $\mathrm{H}_{2} \mathrm{O}_{2}$ in the tumor tissue significantly limits the CDT efficacy. To cope with these challenges, Sun et al. [14] constructed a liposomal formulation composed of dichloroacetic acid (DCA) and MOF-Fe ${ }^{2+}$ (MD@Lip). As an acetic acid derivative, DCA does not only enhance the intratumor acidity but also promotes glucose oxidation to produce $\mathrm{H}_{2} \mathrm{O}_{2}$ in mitochondria. The in vitro and in vivo experiments demonstrated that the $\mathrm{CDT}$ efficacy was significantly improved by the synergistic effect of DCA and $\mathrm{MOF}-\mathrm{Fe}^{2+}$. In addition to DCA, glucose oxidase (GOx) is often used to increase the contents of $\mathrm{H}^{+}$and $\mathrm{H}_{2} \mathrm{O}_{2}$ by catalyzing glucose oxidation in the presence of $\mathrm{O}_{2}$. Accordingly, Fang et al. [15] fabricated a cascade enzymatic/Fenton catalytic reaction by incorporating GOx into a Co-ferrocene MOF. GOx was then activated by intracellular glucose to generate gluconic acid and $\mathrm{H}_{2} \mathrm{O}_{2}$, which effectively increased. $\mathrm{OH}$ production through $\mathrm{Fe}^{2+}$-mediated Fenton reaction. However, the limited concentration of $\mathrm{O}_{2}$ in the tumor tissue impaired the catalytic activity of GOx and hindered CDT efficacy. Considering the influence of temperature for an effective Fenton reaction, they fabricated a $\mathrm{Zr}$-ferrocene MOF nanosheet using a $\mathrm{Zr}-\mathrm{O}$ cluster and 1,1'-ferrocenedicarboxylic acid, which possessed excellent photothermal conversion efficiency [16]. Given the elevated temperature induced by photothermal therapy (PTT), Zr-ferro- cene MOF exhibited excellent CDT effects against the tumor owing to the increased $\cdot \mathrm{OH}$ yield. The as-prepared $\mathrm{Zr}$-ferrocene MOF is an "all-in-one" synergistic therapy for CDT and PTT that does not require any other auxiliary agents. In addition to directly delivering $\mathrm{Fe}^{2+}$, certain studies demonstrated that $\mathrm{Fe}^{3+}$ can be reduced into $\mathrm{Fe}^{2+}$ and catalyze the Fenton reaction to conduct CDT. For example, Gong et al. [17] synthesized a Hf-based MOF modified with $\mathrm{Fe}^{3+}$ (Hf-BPY-Fe), which promoted the conversion of $\mathrm{Fe}^{3+}$ to $\mathrm{Fe}^{2+}$ by enriched electrons after $\mathrm{X}$-ray irradiation and ultimately promoted the Fenton reaction to produce $\cdot \mathrm{OH}$. As shown in Fig. 2, this study provides a new strategy to simultaneously use the metal ion and organic linker in MOFs for realizing the synergistic therapy of CDT and radiotherapy.

Zhang et al. [18] fabricated a nanoamplifier by loading GOx into a $\mathrm{Fe}^{3+}$-based MOF (MIL-100) and coating it with hyaluronic acid (HA). The as-prepared nanoamplifier had an excellent theranostic effect against tumor owing to the synergistic amplification effect of GOx and MIL-100. Subsequently, they reported that MIL-100 can simultaneously serve as a carrier for 2,2'-azino-bis(3ethylbenzothiazoline-6-sulfonic acid) (ABTS) and a peroxidase-like nanozyme [19]. The fabricated ABTS@MIL100/PVP (AMP) nanoreactors achieved the combined therapy of enhanced CDT (ECDT) and PTT using a "twostep rocket launching-like" process (Fig. 3). Recently, Xue et al. [20] constructed DMH nanoparticles composed of MIL-100, doxorubicin (DOX), and an HA coating. The HA coating enabled the DMH nanoparticles to target tumor cells. Then, the loaded DOX combined with the -OH generated from the Fenton-like reaction significantly enhanced the antitumor efficiency.

In addition to Fe-containing catalysts, other metal ions, 


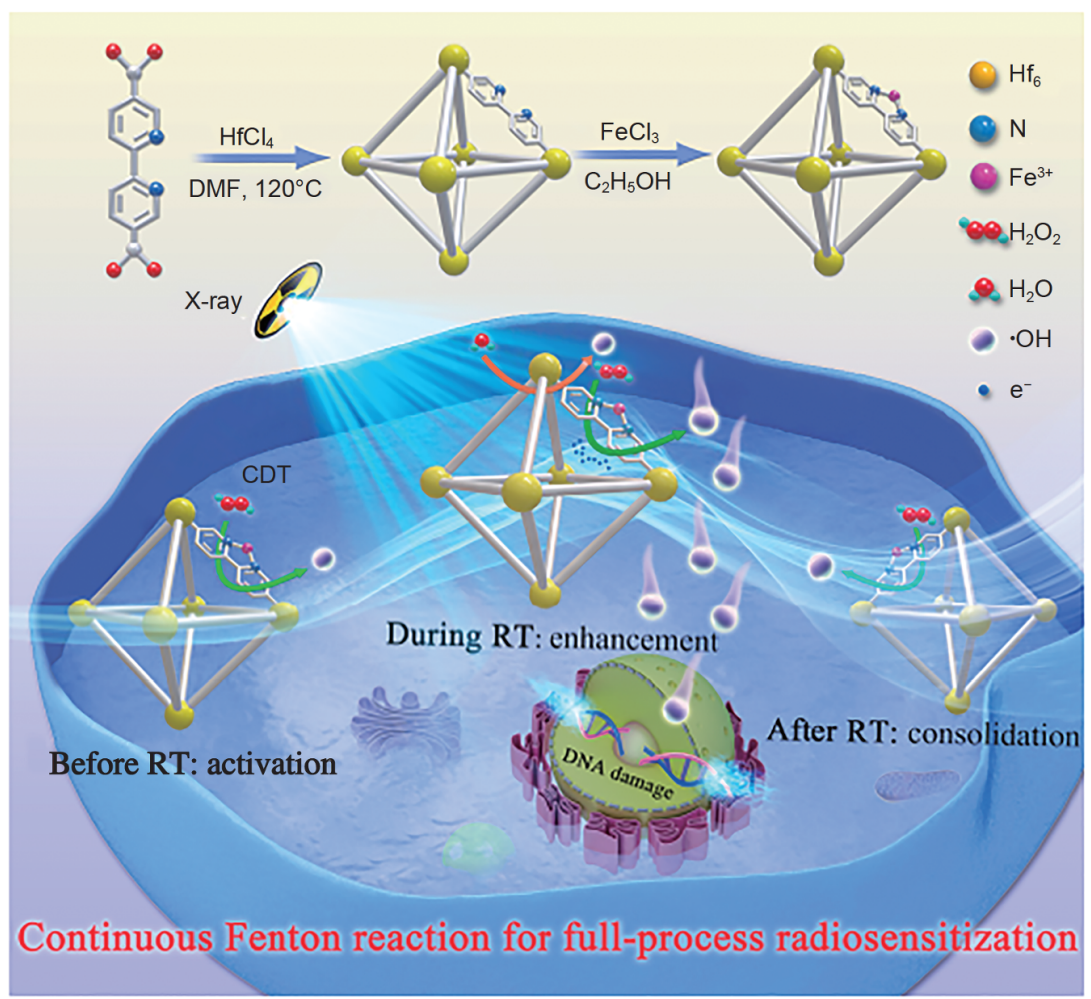

Figure 2 Schematic of the synthesis of Hf-BPY-Fe and its anti-tumor mechanism. Reproduced with permission from ref. [17], Copyright 2020, American Chemical Society.

a

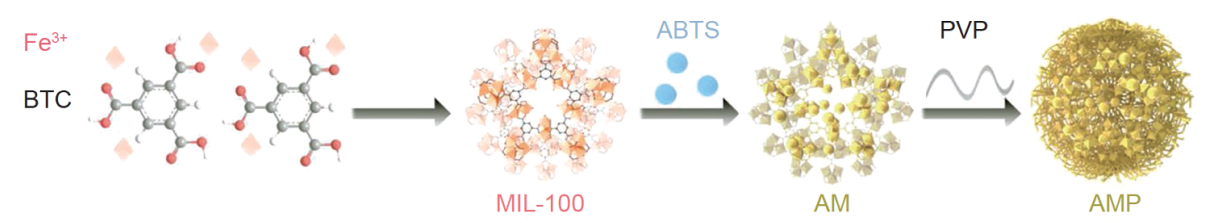

b

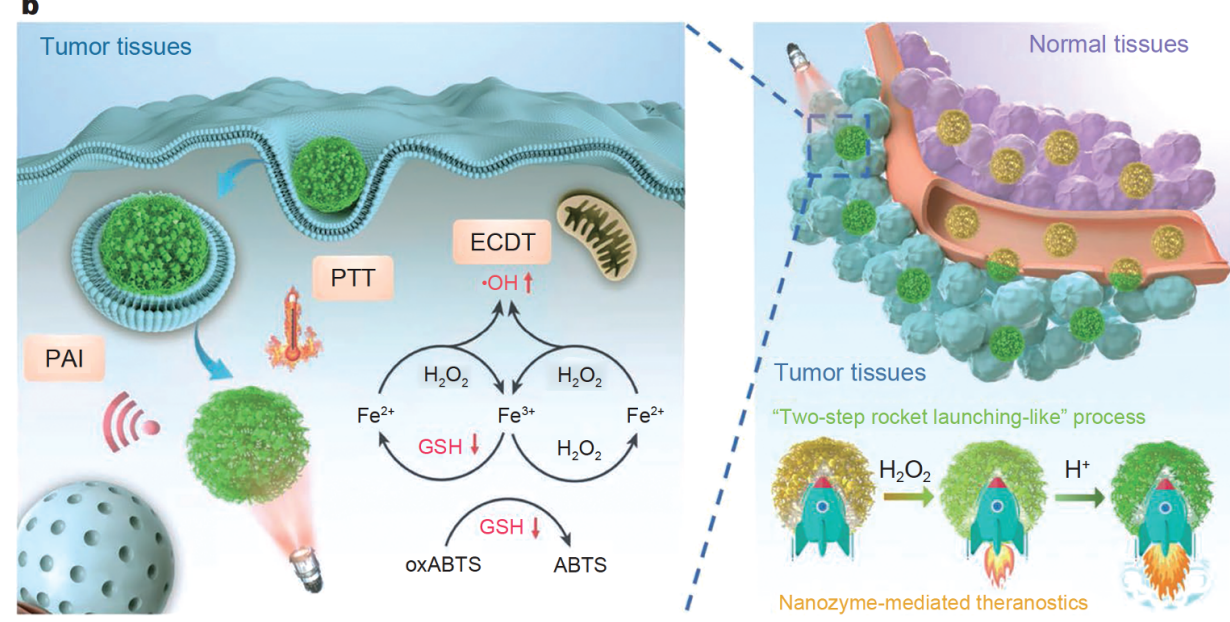

Figure 3 Schematic of the synthesis of AMP and its anti-tumor mechanism. BTC stands for benzenetricarboxylic acid; AM represents ABTS@MIL100; PAI stands for photoacoustic imaging. Reproduced with permission from ref. [19], Copyright 2019, John Wiley \& Sons Inc. 
such as $\mathrm{Cu}^{2+}, \mathrm{Cu}^{+}, \mathrm{Co}^{2+}$ and $\mathrm{Mn}^{2+}$, can trigger a Fentonlike reaction to produce $\cdot \mathrm{OH}$. Therefore, other metalbased MOFs can serve as a catalyst for initiating a CDT process. For example, $\mathrm{Ni}$ et al. [21] synthesized $\mathrm{Cu}^{2+}$-based MOFs using 5,10,15,20-tetrabenzoatoporphyrin (TBP) as a ligand (Cu-TBP). The $\mathrm{pH}-$ sensitive $\mathrm{Cu}$-TBP was then internalized in cells and decomposed to $\mathrm{Cu}^{2+}$ and TBP, which could be used for CDT and PDT, respectively. Moreover, the synergistic effect of $\mathrm{Cu}$-TBP-mediated radical treatment and anti-PD-L1 immunotherapy effectively inhibited the growth of distant tumors in B16F10 tumor-bearing mice. Recently, Wang et al. [22] incorporated GOx into a $\mathrm{Cu}^{2+}$-based MOF (PCN224), which was then coated with $\mathrm{MnO}_{2}$ to avoid GOx damaging normal cells. $\mathrm{MnO}_{2}$ then reacted with the endogenous $\mathrm{H}_{2} \mathrm{O}_{2}$ to generate $\mathrm{O}_{2}$, which promoted the catalytic oxidation of glucose by GOx to produce abundant $\mathrm{H}_{2} \mathrm{O}_{2}$ for CDT. The released $\mathrm{Cu}^{2+}$ from PCN-224 consumed the intratumor glutathione (GSH) and was converted into $\mathrm{Cu}^{+}$. They demonstrated that $\mathrm{Cu}^{+}$synchronously induced the production of $\cdot \mathrm{OH}$ and ${ }^{1} \mathrm{O}_{2}$ through a Fenton-like reaction and the Russell mechanism, respectively. These results reveal a potential new research direction for nanocatalytic therapy based on the Russell mechanism. Compared with the Cu-based MOF, a
Co-based zeolitic imidazolate framework (ZIF-67) has improved acidic responsiveness, which enables the faster release of $\mathrm{Co}^{2+}$ to induce a Fenton-like reaction. Thus, we developed a nanocatalytic medicine $\left(\mathrm{CaO}_{2} @ \mathrm{DOX} @ Z I F-\right.$ 67) by encapsulating DOX and calcium peroxide $\left(\mathrm{CaO}_{2}\right)$ within ZIF-67 [23]. In the acidic TME, ZIF-67 was broken down to release $\mathrm{Co}^{2+}$ and DOX. Then, the exposed $\mathrm{CaO}_{2}$ reacted with $\mathrm{H}_{2} \mathrm{O}$ to generate both $\mathrm{O}_{2}$ and $\mathrm{H}_{2} \mathrm{O}_{2}$ [24]. The generated $\mathrm{H}_{2} \mathrm{O}_{2}$ promoted a Co ${ }^{2+}$-based Fenton-like reaction that generated $\cdot \mathrm{OH}$; however, the generated $\mathrm{O}_{2}$ relieved tumor hypoxia and enhanced the DOX efficacy (Fig. 4). This simple design can simultaneously enhance the efficacy of CDT and chemotherapy. Loading a Fenton or Fenton-like reaction catalyst into an MOF is another approach to initiate CDT. Considering high loading capacity and acid sensitivity, Ranji-Burachaloo et al. [25] used a Zn-based MOF (ZIF-8) as a carrier to simultaneously deliver GOx and natural hemoglobin to tumor tissue. The pH-sensitive ZIF-8 released GOx and hemoglobin into the tumor tissue under the acidic TME. GOx then consumed glucose to produce gluconic acid and $\mathrm{H}_{2} \mathrm{O}_{2}$. Furthermore, the released hemoglobin provided $\mathrm{Fe}^{2+}$ to catalyze the Fenton reaction. Because of the increased acidity and $\mathrm{H}_{2} \mathrm{O}_{2}$ content in the tumor tissue, the CDT efficacy was considerably enhanced. Recently,

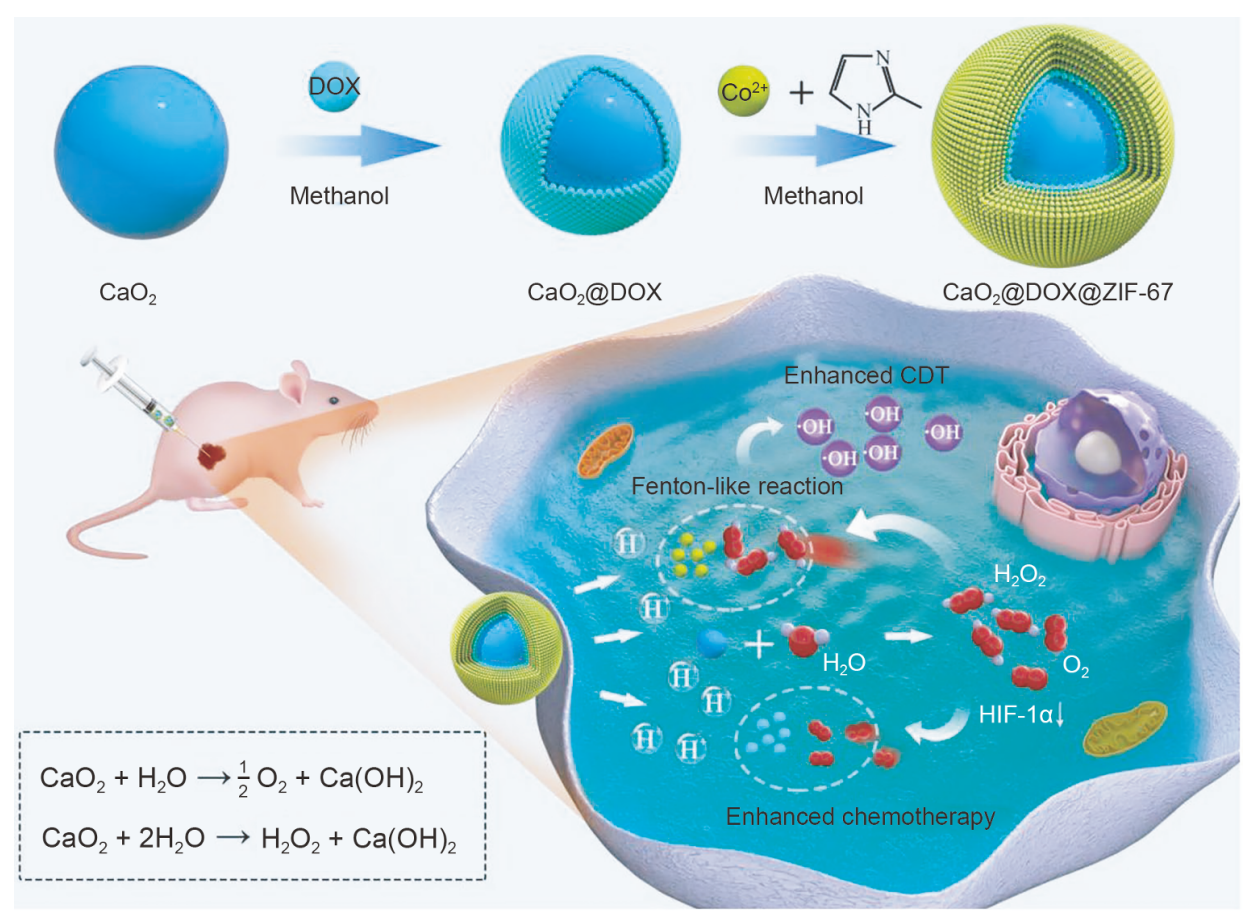

Figure 4 Schematic of the synthesis of $\mathrm{CaO}_{2} @$ DOX@ZIF-67 and its anti-tumor mechanism. Reproduced with permission from ref. [23], Copyright 2019, John Wiley \& Sons Inc. 
Xie et al. [26] synthesized a double-layer ZIF-8 construction termed as $\mathrm{O}_{2}$-Cu/ZIF-8@Ce6/ZIF-8@F127 (OCZCF) to co-deliver $\mathrm{O}_{2}, \mathrm{Cu}^{2+}$, and chlorin e6 (Ce6). $\mathrm{Cu}^{2+}$ both doubled the $\mathrm{O}_{2}$ loading capacity of ZIF- 8 and catalyzed the Fenton-like reaction after being reduced by GSH. Therefore, OCZCF realized the enhanced synergetic $\mathrm{PDT}$ and CDT by relieving hypoxia and consuming GSH.

Recently, the application of MOFs as a CDT has attracted considerable attention. In this review, we summarize the recent developments in the application of MOFs in CDT. Most studies utilize the $\mathrm{pH}$ sensitivity of MOFs, which can provide usable metal ions for the Fenton or Fenton-like reaction. Moreover, the well-defined pores of MOFs can be used to deliver other therapeutic agents, which can enhance CDT or allow for the development of combined treatment strategies. Therefore, MOFs are a class of promising nanocatalytic medicine. Although the application of MOFs in CDT has yielded encouraging results, certain challenges remain. First, multiple studies demonstrate that MOFs have unfavorable properties such as poor water solubility and poor stability. Poor water solubility leads to severe aggregation and increases the toxic side effects; however, poor stability leads to premature decomposition during circulation. Thus, additional efforts should be made to prepare MOFs that are hydrophilic and stable. Secondly, certain studies reported that MOFs show minimal catalytic activity under physiological conditions; thus, auxiliary reagents become indispensable, which complicates the preparation of MOFs-based nanocatalytic medicine further. Therefore, developing a simple method for integrating multifunction into MOF-based nanocatalytic medicine is desirable. Thirdly, although MOFs can deliver more Fenton or Fenton-like catalysts to a tumor to enhance CDT efficacy, the retention of these heavy metal ions in the body may have potential toxic side effects. Furthermore, toxicity induced by organic linkers is another problem. Construction of MOF-based nanocatalytic medicine using endogenous or biosafe organic linkers and metal ions is a preferable method to meet this challenge. MOF-based nanocatalytic medicine is still in its infancy; thus, the development of new strategies to overcome these obstacles would expand the application prospects of MOF-based nanocatalytic medicine in CDT. We believe that MOFs have considerable potential in improving the CDT efficacy.

Received 9 July 2020; accepted 8 September 2020; published online 13 October 2020

1 Jasim KA, Gesquiere AJ. Ultrastable and biofunctionalizable con- jugated polymer nanoparticles with encapsulated iron for ferroptosis assisted chemodynamic therapy. Mol Pharm, 2019, 16: 48524866

2 Lin $\mathrm{H}$, Chen Y, Shi J. Nanoparticle-triggered in situ catalytic chemical reactions for tumour-specific therapy. Chem Soc Rev, 2018, 47: 1938-1958

3 Yang B, Chen Y, Shi J. Nanocatalytic medicine. Adv Mater, 2019, 31: 1901778

4 Wang J, Wang Y, Wang R, et al. Targeted nanoparticles for precise cancer therapy. Sci China Life Sci, 2019, 62: 1392-1395

5 Fan W, Qi Y, Wang R, et al. Calcium carbonate-methylene blue nanohybrids for photodynamic therapy and ultrasound imaging. Sci China Life Sci, 2018, 61: 483-491

6 Qian X, Zhang J, Gu Z, et al. Nanocatalysts-augmented Fenton chemical reaction for nanocatalytic tumor therapy. Biomaterials, 2019, 211: 1-13

7 Pan X, Wang H, Wang S, et al. Sonodynamic therapy (SDT): a novel strategy for cancer nanotheranostics. Sci China Life Sci, 2018, 61: 415-426

8 Jiang K, Zhang L, Xia T, et al. A water-stable fcu-MOF material with exposed amino groups for the multi-functional separation of small molecules. Sci China Mater, 2019, 62: 1315-1322

$9 \mathrm{He} \mathrm{J,} \mathrm{Xu} \mathrm{J,} \mathrm{Yin} \mathrm{J,} \mathrm{et} \mathrm{al.} \mathrm{Recent} \mathrm{advances} \mathrm{in} \mathrm{luminescent} \mathrm{metal-}$ organic frameworks for chemical sensors. Sci China Mater, 2019, 62: $1655-1678$

10 Wang Y, Li Y, Wang Z, et al. Reticular chemistry in electrochemical carbon dioxide reduction. Sci China Mater, 2020, 63: 1113-1141

11 Sun C, Chang L, Hou K, et al. Encapsulation of live cells by metalorganic frameworks for viability protection. Sci China Mater, 2019, 62: $885-891$

12 Cai W, Wang J, Chu C, et al. Metal-organic framework-based stimuli-responsive systems for drug delivery. Adv Sci, 2019, 6: 1801526

13 Ranji-Burachaloo H, Karimi F, Xie K, et al. MOF-mediated destruction of cancer using the cell's own hydrogen peroxide. ACS Appl Mater Interfaces, 2017, 9: 33599-33608

14 Sun L, Xu Y, Gao Y, et al. Synergistic amplification of oxidative stress-mediated antitumor activity via liposomal dichloroacetic acid and MOF-Fe ${ }^{2+}$. Small, 2019, 15: 1901156

15 Fang C, Deng Z, Cao G, et al. Co-ferrocene MOF/glucose oxidase as cascade nanozyme for effective tumor therapy. Adv Funct Mater, 2020, 30: 1910085

16 Deng Z, Fang C, Ma X, et al. One stone two birds: Zr-Fc metalorganic framework nanosheet for synergistic photothermal and chemodynamic cancer therapy. ACS Appl Mater Interfaces, 2020, 12: 20321-20330

17 Gong T, Li Y, Lv B, et al. Full-process radiosensitization based on nanoscale metal-organic frameworks. ACS Nano, 2020, 14: 30323040

18 Zhang Y, Lin L, Liu L, et al. Positive feedback nanoamplifier responded to tumor microenvironments for self-enhanced tumor imaging and therapy. Biomaterials, 2019, 216: 119255

19 Liu F, Lin L, Zhang Y, et al. A tumor-microenvironment-activated nanozymes-mediated theranostic nanoreactor for imaging-guided combined tumor therapy. Adv Mater, 2019, 31: 1902885

20 Xue T, Xu C, Wang Y, et al. Doxorubicin-loaded nanoscale metalorganic framework for tumor-targeting combined chemotherapy and chemodynamic therapy. Biomater Sci, 2019, 7: 4615-4623

$21 \mathrm{Ni} \mathrm{K}$, Aung T, Li S, et al. Nanoscale metal-organic framework 
mediates radical therapy to enhance cancer immunotherapy. Chem, 2019, 5: 1892-1913

22 Wang Z, Liu B, Sun Q, et al. Fusiform-like copper(ii)-based metalorganic framework through relief hypoxia and GSH-depletion Coenhanced starvation and chemodynamic synergetic cancer therapy. ACS Appl Mater Interfaces, 2020, 12: 17254-17267

23 Gao S, Jin $\mathrm{Y}, \mathrm{Ge} \mathrm{K}$, et al. Self-supply of $\mathrm{O}_{2}$ and $\mathrm{H}_{2} \mathrm{O}_{2}$ by a nanocatalytic medicine to enhance combined chemo/chemodynamic therapy. Adv Sci, 2019, 6: 1902137

24 Gao S, Fan M, Li Z, et al. Smart calcium peroxide with self-sufficience for biomedicine. Sci China Life Sci, 2020, 63: 152-156

25 Ranji-Burachaloo H, Reyhani A, Gurr PA, et al. Combined Fenton and starvation therapies using hemoglobin and glucose oxidase. Nanoscale, 2019, 11: 5705-5716

26 Xie Z, Liang S, Cai X, et al. O-Cu/ZIF-8@Ce6/ZIF-8@F127 composite as a tumor microenvironment-responsive nanoplatform with enhanced photo-/chemodynamic antitumor efficacy. ACS Appl Mater Interfaces, 2019, 11: 31671-31680

Acknowledgements This work was supported by the National Natural Science Foundation of China (31971304 and 21601046), the Natural Science Key Foundation of Hebei Province (B2017201226), and the Doctoral Candidate Innovation Funding Project of Hebei Province (CXZZBS2020022).

Author contributions Gao S and Han Y wrote the paper with support from Zhang J. All authors contributed to the general discussion.

Conflict of interest The authors declare that they have no conflict of interest.



Shutao Gao received his $\mathrm{PhD}$ degree in 2020 from Hebei University under the direction of Professor Jinchao Zhang. He is now a professor of Hebei Agricultural University. His main research interests are the preparation and applications of functional nanomaterials.

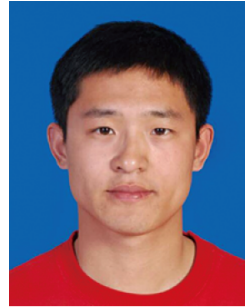

Yu Han received his BSc degree from Hebei University in 2015. He is now a PhD student of Hebei University. His research interests are nanomedicine and molecular diagnosis.



Jinchao Zhang received his $\mathrm{PhD}$ from Zhejiang University in 2001. He did postdoctoral research at Peking University from 2001 to 2003. Then, he worked as a senior visiting scholar at the City University of Hong Kong for three years. Currently, Dr. Zhang is a Professor at Hebei University. His research interests focus on the design and syntheses of nanomaterials for cancer diagnosis and therapy.

\section{金属-有机框架纳米催化药物在化学动力学中的 应用}

高书涛 ${ }^{1,2 \dagger}$, 韩宇 $^{2 \dagger}$, 樊沝 ${ }^{2}$, 李振华 ${ }^{2}$, 葛昆 $^{2}$, 梁兴杰 $^{3}$, 张金超 $^{2^{*}}$

摘要 化学动力学疗法是一种基于芬顿或类芬顿反应原理的肿瘤 纳米催化治疗方式, 已成为一种极具发展潜力的肿瘤治疗策略. 金 属-有机框架材料, 因具有比表面积大、孔隙度高、负载能力强、 酸响应性能好等优点, 已被用于合成各种纳米催化药物. 本文总结 了近年来基于金属-有机框架材料的纳米催化药物的设计、合成策 略, 分析并展望了其用于肿瘤化学动力学治疗所面临的问题和未 来的发展趋势. 